\title{
Repeated Generalized Seizures Induce Time-Dependent Changes in the Behavioral Seizure Response Independent of Continued Seizure Induction
}

\author{
Gary M. Samoriski and Craig D. Applegate \\ Program in Neuroscience and The Comprehensive Epilepsy Program, University of Rochester School of Medicine and \\ Dentistry, Rochester, New York 14642
}

\begin{abstract}
This study examined both the acute and long-lasting changes in seizure susceptibility that occur in response to the repeated induction of generalized seizure activity. Daily flurothyl-induced generalized clonic seizures resulted in a progressive decrease in both the generalized seizure threshold and the latency to the first myoclonic jerk. The threshold reduction was significant as early as the second trial and was maximal by trial 5 . However, a minimum of eight seizures was necessary for the maximal reduction to be long-lasting. The present study also examined the effects of the number of seizures and the duration of the stimulation-free interval on the type of generalized seizure expressed. During the induction phase of the experiment, only generalized clonic seizures ("forebrain seizures") were expressed. If, however, the animal was retested after a 1, 2, 3, or 4 week stimulation-free interval, a progressive increase in both the proportion of animals expressing "brainstem seizure" be-
\end{abstract}

haviors and the median seizure score was observed. The progression of flurothyl-induced generalized seizure behaviors was significantly altered if (1) a minimum of eight generalized clonic seizures had been expressed, and (2) a minimum of a 2 week stimulation-free interval followed. Fewer generalized clonic seizures failed to reliably produce changes in seizure phenotype, even after extended stimulus-free intervals. These data indicate that specific kindling processes are initiated during the interval of repeated seizure induction and evolve in the absence of continued seizure induction. Furthermore, these mechanisms of epileptogenesis were found to be manifest predominantly as a change in the seizure phenotype expressed and to proceed independent of changes in the generalized seizure threshold.

Key words: epileptogenesis; generalized clonic seizure; generalized tonic seizure; kindling; flurothyl; mouse
Epileptogenesis, in experimental animals, is characterized by a decrease in the generalized seizure threshold (GST) and a change in the motor seizure behavior expressed in response to repeated exposure to a stimulus of constant magnitude. The neural reorganization that underlies the enhanced seizure susceptibility progressively develops in response to the repeated induction of either focal or generalized seizure activity (Goddard et al., 1969; Ramer and Pinel, 1976). Once established, the modified seizure state is maintained for at least 12 weeks and perhaps longer (Dennison et al., 1995). Collectively, these phenomena are the hallmarks of kindling. Although the kindled state is established as a result of alterations effected during the course of seizure induction, it is unknown whether these changes continue to evolve in the absence of exogenous input. In this report, we present evidence demonstrating that repeated generalized ictal activity initiates changes in neural function that are time-dependent, evolve independent of continued seizure induction, and are manifest as a change in the phenotype of the expressed motor seizure.

Received Aug. 23, 1996; revised April 21, 1997; accepted April 24, 1997.

This work was supported by National Institutes of Health Grant NS26865 to C.D.A.

Preliminary results of this study have been published in abstract form at the 24th Annual Meeting, Society for Neuroscience, 1994.

Correspondence should be addressed to Prof. Craig D. Applegate, Box 605, University of Rochester School of Medicine and Dentistry, 601 Elmwood Avenue, Rochester, NY 14642

Dr. Samoriski's present address: Department of Pharmacology and Physiology, Box 711, University of Rochester School of Medicine and Dentistry, 601 Elmwood Avenue, Rochester, NY 14642.

Copyright (C) 1997 Society for Neuroscience $0270-6474 / 97 / 175581-10 \$ 05.00 / 0$
The goal of the present study was to characterize this phenomenon in mice using the inhalant chemoconvulsant flurothyl. The administration of flurothyl has been shown to reliably produce generalized seizure behaviors in rats and mice (Truitt et al., 1960; Adler et al., 1967; Prichard et al., 1969). Clonic seizure behaviors are exhibited if the exposure is terminated with the beginning of a sustained generalized seizure. Although repeated flurothyl testing has been shown to result in a progressive decrease in the seizure threshold (Adler et al., 1967; Prichard et al., 1969), the effect of multiple flurothyl-induced generalized seizures on the behavioral seizure response has not been well described. In addition, long-lasting changes that may result from repeated flurothyl testing have not been documented. Consequently, this study was designed to describe the long-term effects of repeated seizure testing with flurothyl in terms of (1) the number of seizures necessary to initiate mechanisms responsible for changes in seizure susceptibility, and (2) the minimum duration of the stimulation-free interval before the change in seizure phenotype is observed.

\section{MATERIALS AND METHODS}

Animals. Adult (6 weeks of age) male C57BL/6J mice were obtained from Jackson Laboratories (Bar Harbor, ME). An adaptational period (minimum 1 week) was allowed before the beginning of seizure testing. The animals were grouped in a temperature-controlled environment under a 12:12 hr light/dark cycle (lights on at 6:00 A.M.). Food and water were provided ad libitum. All animal use procedures were in accordance with the National Institutes of Health Guide for the Care and Use of Laboratory Animals (1985). 


\section{Experimental design}

Rationale. Flurothyl, administered by inhalation, was chosen to study the effects of repeated generalized seizures on CNS function. In contrast to other chemoconvulsants that are administered systemically (intraperitoneally or intravenously), once sustained flurothyl-induced seizure activity begins, the investigator can control the duration of action of the drug by opening the chamber to room air. Because flurothyl does not depend on metabolism for its action and is rapidly eliminated by the lungs, opening the chamber essentially terminates its action.

To evaluate the acute and long-term effects of repeated flurothylinduced seizures on the GST in mice, a design was used such that each exposure to flurothyl produced a sustained generalized seizure with predominantly clonic motor manifestations. Generalized seizures were elicited by a $10 \%$ solution of flurothyl and repeated once every $24 \mathrm{hr}$. Previous studies have shown that this is an effective paradigm for eliciting multiple seizures with low mortality and results in progressively lower thresholds without significantly changing the motor characteristics of the seizure (Adler et al., 1967; Prichard et al., 1969). In addition, the fact that the decrease in GST asymptotes with repeated testing indicates that a maximal and stable change in seizure threshold can be achieved under these conditions (Adler et al., 1967; Prichard et al., 1969). Based on these observations, two criteria were used to define the seizure threshold asymptote: (1) when the GST had become significantly different compared with the naive state (first trial), and (2) when the threshold was sustained and unchanging for at least four consecutive trials (i.e., no significant difference among any of the four trials). Preliminary experiments revealed that eight trials satisfied these criteria. These animals then went unstimulated for 4 weeks and were subsequently retested with flurothyl to assess whether the change in threshold was long-lasting. The duration of the stimulus-free interval was chosen, because the retention of the kindling effect at 1 month is generally accepted as a measure of permanence. However, on retesting at $28 \mathrm{~d}$, we observed a significant change in the behavioral seizure response of the animal. Therefore, the following experiments were designed to characterize the minimum requirements necessary to elicit acute and long-term changes in the seizure susceptibility, as measured by latency to the sustained motor event (GST) and phenotype (behavioral seizure response).

Treatment. Preliminary data indicated that when preceded by eight generalized clonic seizures, a 4 week stimulus-free interval was sufficient to effect a significant change in the behavioral seizure response of the animal when retested with flurothyl. To determine whether the altered behavioral response was apparent after a stimulus-free interval of lesser duration, these experiments were repeated and extended to include 1,2, and 3 week intervals. Therefore, generalized seizures were elicited once every $24 \mathrm{hr}$ for $8 \mathrm{~d}$. Separate groups of mice went unchallenged for intervals of $1(n=13), 2(n=24), 3(n=11)$, or $4(n=18)$ weeks, at which time they were retested and the seizure response evaluated. In addition, a subset of animals from the 1,2 , and 3 week groups were rechallenged $50 \mathrm{~d}$ after their retest trial to document whether changes in the behavioral seizure response and GST were maintained at these longer intervals.

Because eight daily generalized clonic seizures (followed by a 4 week stimulus-free interval) were found to result in a near maximal change in the proportion of animals exhibiting a "brainstem seizure," it was of interest to determine whether the elicitation of fewer generalized clonic seizures produced a similar change in the behavioral seizure response. With $24 \mathrm{hr}$ separating the seizures, $1,2,4,6$, or 8 generalized motor convulsions were induced in different groups of mice. The GST and motor seizure characteristics were then reevaluated 4 weeks $(n=5,6,6$, 6 , and 18 , respectively) after the last trial. The eight-trial group was the same as that described above and was used for comparison purposes.

The results of these experiments indicated that fewer than eight generalized seizures did not produce a significant change in the behavioral seizure response when retested after a 4 week stimulus-free interval. Therefore, we evaluated whether fewer than eight generalized seizures required a longer stimulus-free interval for the change in the behavioral seizure response to become apparent. Consequently, 2 ( $n=$ $6)$ or $4(n=6)$ generalized clonic seizures were elicited in separate groups of mice that were then retested after an 8 week stimulus-free interval.

Finally, to determine whether similar changes in the behavioral seizure response occurred without an intervening stimulus-free interval, a separate group of mice $(n=6)$ was tested once every $24 \mathrm{hr}$ for 21 consecutive days to characterize the effects of continuous testing on the behavioral seizure response. The behavioral profile of this group was compared with animals that had exhibited eight daily seizures followed by either a 1 or 2 week stimulus-free interval.

\section{Seizure induction and quantitation}

After a $2 \mathrm{hr}$ acclimatization period after transport to the laboratory from the animal facility, mice were individually placed in a 2.4 liter closed Plexiglass chamber. Generalized seizures were elicited using a $10 \%$ solution of flurothyl (2,2,2-trifluoroethyl ether, Aldrich Chemical, Milwaukee, WI) in $95 \%$ ethanol. Flurothyl was administered by infusion $(0.15 \mathrm{ml} / \mathrm{min})$ onto a gauze pad (changed after each exposure) suspended at the top of the chamber using a $20 \mathrm{ml}$ B-D Multifit syringe driven by a Sage inf usion pump (Sage Instruments, White Plains, NY). Inf usion was terminated and the chamber opened to room air with the onset of sustained generalized seizure activity. Onset of a generalized seizure was defined as a sustained loss of postural control $(>2 \mathrm{sec})$. Seizure tests were conducted between 8:00 A.M. and 10:00 A.M. to minimize circadian influences on flurothyl-induced seizure thresholds (Davis and Webb, 1963). Latencies (in seconds) from the start of infusion to the onset of each seizure behavior were recorded and used to define the convulsive threshold for each motor behavior expressed. The duration of the total sustained generalized seizure and of each component of the motor seizure was also noted. In all cases, the number of myoclonic jerks was recorded.

\section{Behavioral rating of flurothyl-induced seizures}

To quantify changes in the convulsive pattern expressed, as a function of the number of seizures or the temporal interval between induction of the sensitized state and reexposure to the convulsant stimulus, generalized seizure behaviors were classified using the following behavioral scoring system: grade 1, purely clonic seizure; grade 2, "transitional" behaviors involving high-frequency/low-magnitude bouncing and/or rapid backward motion; grade 3 , running/bouncing episode; grade 4, secondary loss of posture with bilateral forelimb and hindlimb treading; grade 5, secondary loss of posture with bilateral forelimb tonic extension and bilateral hindlimb flexion followed by treading; grade 6, secondary loss of posture with bilateral forelimb and hindlimb tonic extension (THE) followed by treading; grade 7, THE followed by death. The score assigned reflects the highest grade seizure behavior expressed by the animal within a trial. The progression of seizure grades, within this scoring system, reflects specific sets of behaviors expressed by the animal using this paradigm and partially reflects the order in which the behaviors are expressed. This scoring system is not meant to suggest that the magnitude of one behavior is greater than that of another. In all cases, a grade 2 seizure is preceded by a grade 1 seizure. Similarly, grade 3 seizure behaviors are always preceded by a grade 1 seizure, with or without the expression of grade 2 behaviors. Expression of a grade 3 seizure acts as a branch point within the behavior network; that is, the seizure can either end or progress to any one of the grades 4-7 behaviors, but never a combination of the grade 4-7 behaviors.

Because running/bouncing seizures and generalized tonic seizure manifestations can be elicited in the absence of forebrain connections (Kreindler et al., 1958; Browning and Nelson, 1986), these seizure behaviors have been collectively referred to as "brainstem seizures." Therefore, generalized seizures featuring any of the motor behaviors defined above as grades 3-7 are designated "brainstem seizures." Similarly, grades 1-2 seizure behaviors are designated "forebrain seizures," because these behaviors are not expressed after high mesencephalic transection (Magistris et al., 1988; Browning et al., 1993). In addition, myoclonic jerks (MJ), although not a part of this seizure-grading system, are also eliminated after transection. Therefore, this early seizure manifestation is considered to be a component of forebrain seizure activity, but is dealt with separately in the behavioral analysis.

\section{Data analysis}

Animals exhibiting one or multiple generalized seizures during the induction phase were treated as independent groups and distinguished by the duration of the stimulus-free interval or the number of seizures exhibited before the interval that preceded the retest trial. Inclusion of an animal within a particular group in any experiment was by random selection.

To characterize the acute changes in seizure threshold that occur in response to the repeated induction of generalized seizure activity and to determine the effect of treatment on the long-lasting nature of these changes, latencies to the first MJ or the generalized motor event were combined on a per-trial basis to compute a "grand mean" for that seizure 
endpoint for that trial. The grand mean represents all animals in the study (excluding those exhibiting 21 consecutive daily seizures), the total number of which ranged from 101 on trial 1 to 66 by trial 8 . This resulted in a "population" profile for both the threshold to the first MJ and GST. These data were used to construct a curve of best fit defined by a polynomial equation. Before this analysis, a one-way ANOVA was performed on the GST for each trial to confirm that between-group differences did not exist. To evaluate differences between individual trials and to determine when the curves became asymptotic, a one-way ANOVA was performed on the grand mean data and post hoc comparisons made using Scheffe's procedure.

To determine the effect of treatment on the long-lasting nature of the threshold change, the mean latency to the first MJ and generalized seizure for the retest trial of each experimental group was compared with the grand mean, using the regression equation derived from the leastsquares fit. By solving for $x$ in the equation, where $y$ is the measured threshold of the sample, we were able to approximate the location on the curve of the test threshold (retest trial). This allowed us to determine to which trial, within the induction phase, the test threshold was best matched. If, for example, the threshold on the last trial contributed to the asymptotic portion of the regression curve and the threshold on the retest trial was calculated to fall within this portion of the curve, the change in threshold was interpreted to be long-lasting. By contrast, a test threshold estimated to fall within that portion of the regression curve, before it becoming asymptotic, was interpreted as partial or full recovery. Paired $t$ tests were used to describe differences in the GST in a subset of animals that were rechallenged $50 \mathrm{~d}$ after their retest trial.

Differences in the number of animals exhibiting "brainstem seizures" on the retest trial compared with the last trial were determined using the McNemar test; differences between groups were assessed using the $\chi^{2}$ test. The effect of the number of seizures or the duration of the stimulusfree interval on the type of seizure behavior expressed was determined within groups by comparing the behavioral seizure score obtained on the retest trial with the behavioral seizure score of the last trial using the Wilcoxon signed-ranks test. The Spearman rank correlation was used to describe the relationship between the behavioral seizure response and the GST.

\section{RESULTS}

\section{General}

No adverse changes in the health or natural behavior of the animal were observed in the interictal period, in response to either acute or repeated exposure to flurothyl or to flurothylinduced seizures. The animals gained weight characteristic of their age and strain. In addition, the animals were not more aggressive toward either their cage mates or the investigator as a result of the treatment. Importantly, we have exposed $>200$ mice to this experimental paradigm and have never observed a spontaneous ictal event. Mice were in the laboratory daily during flurothyl induction trials and were observed a minimum of every third day during stimulus-free intervals to maintain identification markings. At no time were mice observed to express spontaneous behavioral seizures. In addition, mice exposed to this paradigm are histologically normal at a qualitative level of inspection (our unpublished observations). Thus, the time-dependent changes in seizure susceptibility that occur in response to repeated flurothylinduced seizures (see below) may be driven by distinct processes from those reported in other experimental models in which repeated seizure activity produces widespread histopathology $(\mathrm{Bu}-$ terbaugh and Hudson, 1990; Lothman et al., 1990)

Although most of the animals were 7 or 8 weeks of age at the start of the experiment, a few 9- and 11-week-old animals were used. In general, the difference in age at the beginning of the experiment did not influence the changes in seizure susceptibility that result from the repeated expression of generalized seizures. Although there was a tendency for the GST to be higher on trials 2 and 3 , in 11-week-old animals, no between group differences were observed as a function of age when the GST was combined over the eight trials $\left(F_{(3,523)}=1.53, p=0.21\right)$.

\section{Flurothyl-induced generalized seizure behaviors}

On initial trials (1-3), mice exhibited normal exploratory behaviors that noticeably decreased $\sim 3$ min after the start of flurothyl infusion. The observable reduction in the spontaneous motor activity was restricted to the early trials, because the degree of exploratory activity is less from the outset in later trials. Shortly after the decrease in spontaneous motor activity during early trials, the mouse assumed a posture in which the thoracic and abdominal areas rested on the floor of the chamber, occasionally exhibiting brief intervals of ambulation while maintaining this posture. This posture was rarely observed after the third trial.

The first overt seizure behavior produced by exposure to flurothyl was an $\mathrm{MJ}$ of the head and neck musculature. The number of MJs expressed before the onset of sustained seizure activity was variable, dependent on the seizure history of the animal. Sometimes, particularly in the early trials, a brief burst of forelimb clonus accompanied the MJ episode. Several MJs were expressed before the sustained generalized seizure, the number of which decreased with repeated exposure.

Two patterns of flurothyl-induced generalized seizure behaviors were observed in this study, the expression of which was determined by the seizure history. The first pattern (Fig. 1A), exhibited by naïve animals and animals in which the kindled state had not adequately developed, was defined by the expression of "forebrain seizure" behaviors followed by a period of behavioral postictal depression. "Forebrain seizure" behaviors were characterized by a behavioral response score of grade 1 or grade 2 . Generalized clonic seizures (grade 1) consisted of a sustained loss of postural control, pronounced oro-facial clonus, clonus of the forelimbs and/or hindlimbs, and, in most cases, dorsiflexion of the head. After $\sim 8 \mathrm{sec}$, the animal regained postural control and continued to exhibit facial and forelimb clonus with rearing and, sometimes, falling. Occasionally, these purely clonic behaviors progressed to a qualitatively distinct motor event characterized by high-frequency/low-magnitude bouncing and/or rapid backward motion (grade 2). Using the protocols of this study (see Materials and Methods), the generalized seizure was restricted to these "forebrain seizure" behaviors, after which the animal became postictal. If, however, the animal was exposed continuously to flurothyl vapor, then the postictal period was followed by another series of MJs and, ultimately, the expression of a running/bouncing seizure, with or without the expression of additional "brainstem seizure" behaviors (Fig. 1 $A$ ) (that is, prolonged exposure to flurothyl in naïve mice resulted in both "forebrain" and "brainstem" generalized seizures). The expression of these two seizure types, however, was separated by a significant (minutes) postictal interval.

In contrast, the progression of behaviors, shown in Figure $1 B$, was characteristic of animals in which the minimum requirements for the initiation and development of the kindled state had been exceeded. In these animals, the progression from a "forebrain seizure" to a "brainstem seizure" was not interrupted but was a continuous succession of motor events, even though the chamber had been opened at the start of sustained generalized seizure activity. As in the animals described above, the flurothyl-induced generalized seizure always began with the expression of "forebrain seizure" behaviors. However, instead of becoming behaviorally postictal after the generalized clonic seizure, the animal progressed immediately to a running/bouncing episode (grade 3 ). 


\section{A. Naive}

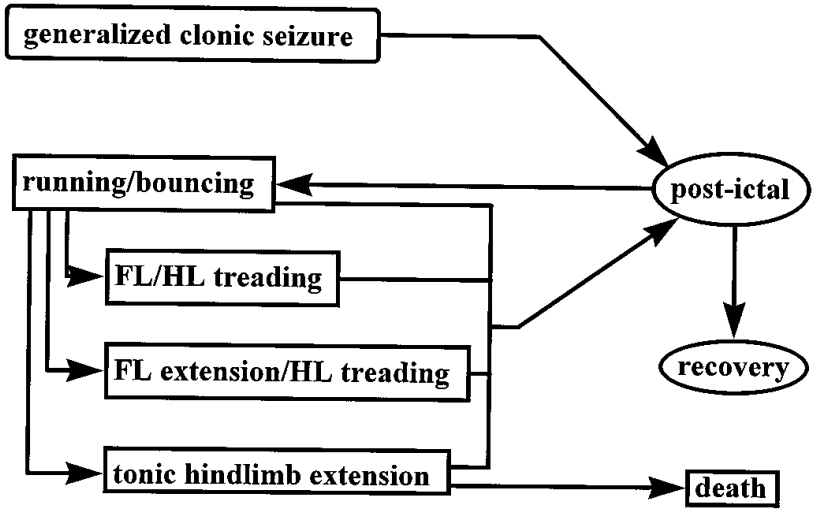

\section{B. Kindled}

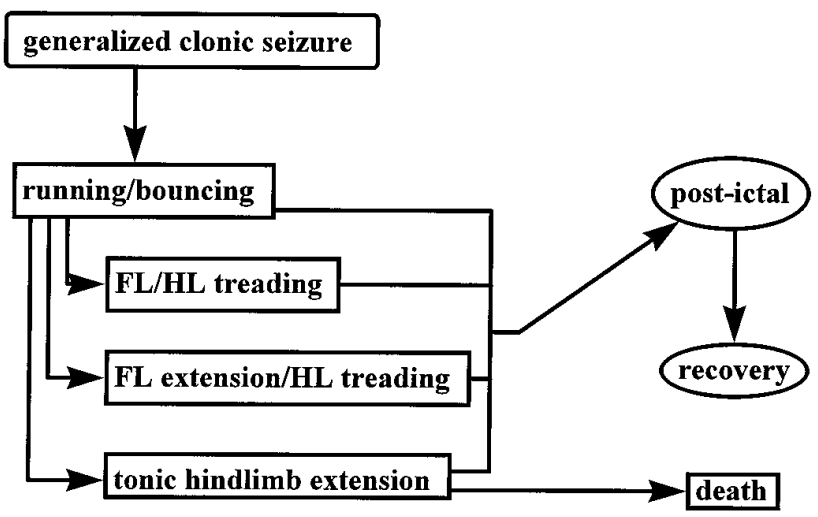

Figure 1. Diagrammatic representation of the behavioral progression of flurothyl-induced generalized seizures. "Forebrain" seizure behaviors are represented in round-cornered boxes, and "brainstem" seizure behaviors are represented in square-cornered boxes. $A$, In naive mice, generalized seizures consist of a generalized clonic seizure that is followed by a period of behavioral postictal depression and recovery. "Brainstem" seizure behaviors are rarely observed using our protocols. However, if flurothyl infusion is continued through the postictal period, naive mice will express seizures with a "brainstem" phenotype. $B$, After the development of flurothyl kindling, there is an uninterrupted progression from a "forebrain" seizure immediately to a "brainstem" seizure, suggesting a facilitated propagation of seizure activity between the two anatomical systems mediating these two generalized seizure phenotypes. $F L$, Forelimb; $H L$, hindlimb.

After expression of the grade 3 behavior, the seizure either ended or continued to evolve uninterrupted into a second loss of postural control followed by either bilateral forelimb/hindlimb treading (grade 4), bilateral tonic extension of the forelimbs and hindlimb treading (grade 5), or bilateral tonic extension of the forelimbs and hindlimbs followed by treading (grade 6). In four cases, expression of THE resulted in the spontaneous death of the animal (grade 7).

Behaviors most often expressed immediately after the end of the overt motor seizure included twitching of the vibrissae and pinnae, head nodding, and periods of digging and gnawing. In addition, animals that expressed "brainstem seizure" behaviors often exhibited a period of "disoriented" ambulation lasting $\sim 20-30 \mathrm{sec}$. In all cases, a period of behavioral postictal depression followed the seizure, the duration of which varied as a function of the expressed seizure phenotype ("brainstem seizures" more than forebrain "seizures").

\section{Threshold effects}

The repeated induction of generalized seizure activity resulted in the progressive reduction in the threshold to the first MJ (Fig. $2 A$ ) and sustained generalized seizure (Fig. 2B). Scheffe's comparisons indicated that the threshold was significantly lower with each trial up to the fourth for the first MJ, and up to the fifth for the GST. The latency to the first MJ on trials 4-8 did not differ significantly among each other. Similarly, the GST was not different among trials 5-8. These data indicate that the decrease in the MJ threshold and the GST was maximal and had reached asymptote by the fourth and fifth trials, respectively.

The number of generalized seizures exhibited before a 4 week stimulus-free interval was found to be an important factor in the long-term maintenance of the threshold reduction. No long-term change in either the MJ threshold or the GST was apparent 4 weeks after the expression of one or two generalized seizures, compared with when the animals left the paradigm. In addition, the acute reduction in the MJ threshold and GST observed, after the expression of four or six generalized seizures, was not longlasting. On retesting, both thresholds partially recovered to a level similar to that observed on trial 2 (Table 1). The same results were obtained if two or four generalized seizures were followed by an 8 week stimulus-free interval. By contrast, eight generalized seizures resulted in both an acute (Fig. $2 A, B$ ) and long-term (Table 1) maximal reduction in both the MJ threshold and the GST.

There appears to be some variability in the maintenance of the threshold reduction after eight generalized seizures for both the first MJ and GST; however, if retested 1, 2, or 4 weeks after the last of the 8 seizures, the reduction in the MJ threshold was apparently maintained, although at 1 week, there appears to be some recovery. By comparison, when animals were retested after a 3 week stimulus-free interval, the observed threshold is equivalent to that of trial 2 (Table 1), indicating a significant rebound of the MJ threshold over time after repeated flurothyl exposure.

The GST exhibited a similar profile. When mice were retested 1,2 , or 4 weeks after the induction of eight daily generalized seizures, the GST corresponded to a trial value of 4.6, 5.2, and 6.6, respectively, based on the regression equation (Fig. 2B, Table 1); that is, the values obtained were within the portion of the curve that is asymptotic. If, however, mice were retested 3 weeks after the last trial, the GST partially recovered to a value equivalent to trial 3 (3.3). This outcome was observed in two independent replications, with groups of animals represented at each time point. The groups that made up a time point were not different on either the last ( 1 week, $t=0.139$, df $=9, p=0.89 ; 2$ weeks, $F_{(2,21)}$ $=0.151, p=0.86 ; 3$ weeks, $t=-0.434, \mathrm{df}=9, p=0.67 ; 4$ weeks, $F_{(2,14)}=3.587, p=0.06$ ) or retest trial (1 week, $t=-0.039, \mathrm{df}=$ $9, p=0.97 ; 2$ weeks, $F_{(2,21)}=0.146, p=0.87 ; 3$ weeks, $t=$ -0.099 , df $=9, p=0.92 ; 4$ weeks, $\left.F_{(2,14)}=0.242, p=0.79\right)$.

The GST also was evaluated in a subset of animals challenged with flurothyl $50 \mathrm{~d}$ after their retest trial. These data demonstrated that although there were small increases in the GST $50 \mathrm{~d}$ after the retest trial compared with the last of eight consecutive daily seizures, the GST remained significantly altered compared with the naive state at all points tested (Fig. $3 A$ ).

\section{Effects on generalized seizure behavior}

The motor behaviors expressed during the eight trials that preceded the stimulus-free interval were almost exclusively clonic, 

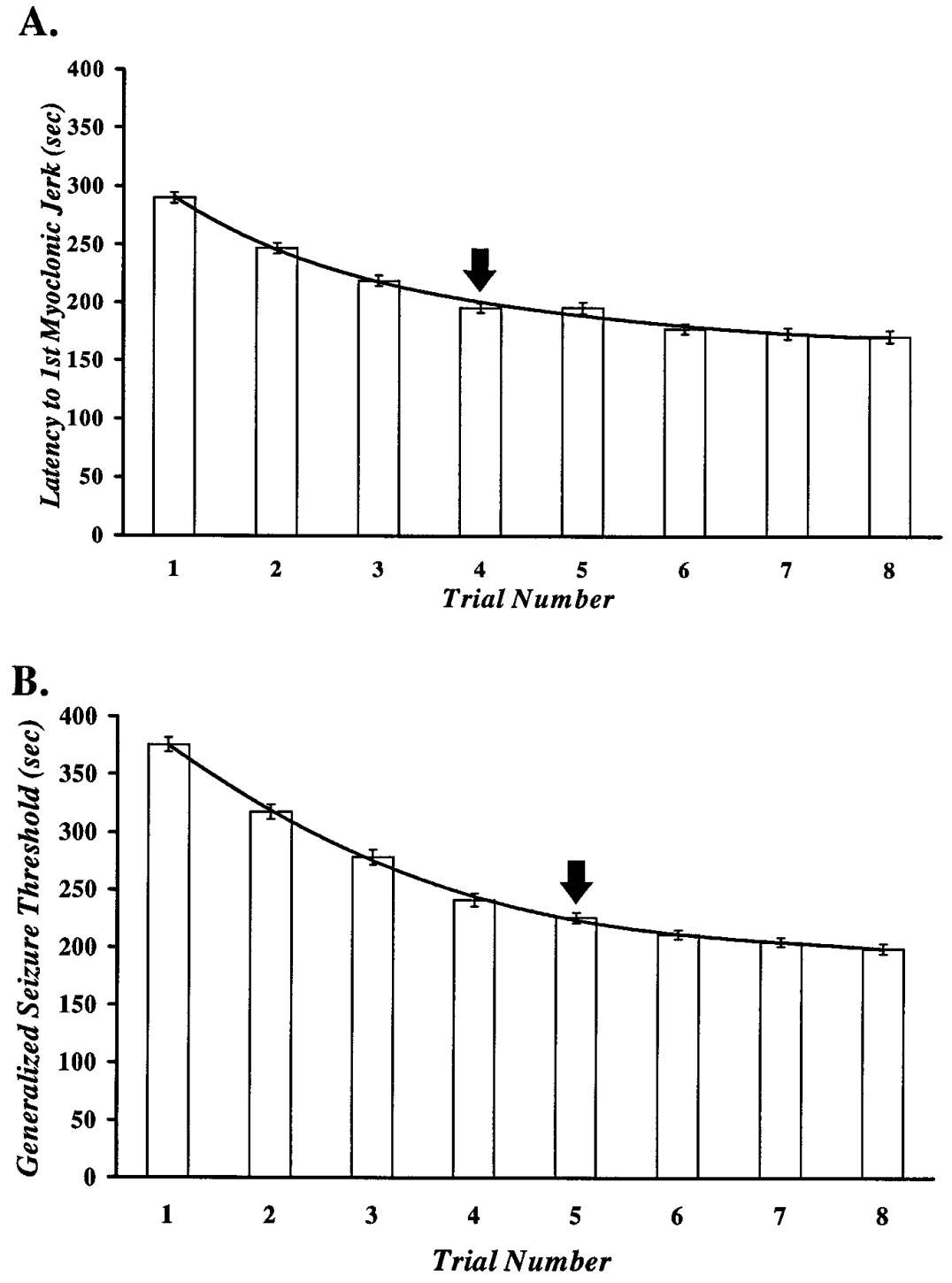

\begin{abstract}
Figure 2. Effect of the repeated induction of flurothylinduced generalized seizures on the threshold to the first MJ $(A)$ and the GST $(B)$ for all mice in this study. The number of animals ranges from between 101 on trial 1 to 66 on trial 8. Both thresholds exhibit a progressive and significant decrease with each trial, up to trial 4 for the first MJ and trial 5 for the GST. The arrows in $A$ and $B$ indicate the point in the induction phase at which the respective threshold becomes asymptotic, indicating a maximal and sustained reduction. Data were evaluated statistically using one-way ANOVA, and comparisons between trials were made using the Scheffe's procedure. The line represents the least-squares fit of the grand mean data. This equation was used to estimate the point in the induction phase with which a sample threshold (on retest) was best matched (cf. Table 1). All values are mean \pm SEM.
\end{abstract}

with a median seizure score of grade 1 . If, however, an animal that exhibited eight seizures went unstimulated for a period of time before a retest trial, these behavioral characteristics were found to be altered as a function of the duration of the stimulusfree interval. After a 1 week stimulus-free interval, no change was observed in either the number of animals exhibiting "brainstem seizures" or the median seizure score expressed when the animal was retested compared with the last trial (Table 2). However, by 2 weeks, a significant increase in both measures was observed. The proportion of mice exhibiting "brainstem" seizures and the median seizure score progressively increased with longer stimulus-free periods (Table 2). Comparisons among groups showed that significantly more animals expressed "brainstem seizures" after either a 2 or a 3 week stimulus-free interval than after a 1 week interval $\left(p<0.05, \chi^{2}\right)$, and that by 4 weeks, this effect was near maximal, with nearly $100 \%$ of animals exhibiting seizure behaviors of grade 3 or above ( $p<0.001$ vs 1 week; $p<0.01$ vs 2 weeks, $\left.\chi^{2}\right)$. No association was found between the GST and the expression of a specific seizure behavior when the animal was retested after the stimulus-free interval (Spearman's $\rho=-0.092$; Fig. 4).

That the observed changes in seizure behavior do not regress and, in fact, continue to evolve with time is illustrated in Figure $3 B$. When rechallenged $50 \mathrm{~d}$ after the retest trial, the median seizure score was found to be increased compared with the eighth trial and the retest trial (Fig. $3 B$ ). Of particular note is the increase in the 1 week group. Whereas the behavioral seizure response was of the "forebrain" type on both the eighth trial and the retest trial, the predominant seizure phenotype was "brainstem" 50 d later (Fig. 3B).

Because the expression of eight daily generalized seizures clearly resulted in an altered behavioral seizure profile, after a 4 week stimulus-free interval, it was of interest to determine whether a similar response could be elicited with fewer seizures followed by an identical stimulus-free interval. One generalized seizure was not sufficient to effect an increase in either the probability of expressing a "brainstem seizure" or the intensity of the seizure behavior. Although some mice that experienced 2, 4, or 6 generalized seizures before the stimulation-free period went on to express "brainstem seizure" behaviors when retested, the proportion was not significantly altered compared with the last trial in each of the three groups (Table 3). By contrast, the induction of eight generalized seizures resulted in a significant increase in the number of animals expressing "brainstem seizure" 
Table 1. Long-term changes in the generalized seizure threshold and the threshold to the first MJ: effect of the number of seizures and the duration of the stimulus-free interval (SFI)

\begin{tabular}{|c|c|c|c|c|c|c|c|c|}
\hline $\begin{array}{l}\text { Trials } \\
+4 \text { wk SFI } \\
\text { (no.) }\end{array}$ & $\begin{array}{l}\text { Measured } \\
\text { threshold } \\
(\mathrm{sec})^{a}\end{array}$ & $\begin{array}{l}\text { Predicted } \\
\text { trial (no.) }{ }^{b}\end{array}$ & $\begin{array}{l}\text { Trials } \\
+8 \text { wk SFI } \\
\text { (no.) }\end{array}$ & $\begin{array}{l}\text { Measured } \\
\text { threshold } \\
(\mathrm{sec})\end{array}$ & $\begin{array}{l}\text { Predicted } \\
\text { trial (no.) }\end{array}$ & $\begin{array}{l}8 \text { Trials } \\
+ \text { weeks SFI }\end{array}$ & $\begin{array}{l}\text { Measured } \\
\text { threshold } \\
(\mathrm{sec})\end{array}$ & $\begin{array}{l}\text { Predicted } \\
\text { trial (no.) }\end{array}$ \\
\hline \multicolumn{9}{|c|}{ Generalized seizure threshold } \\
\hline 1 & $315.8 \pm 10.0$ & 2.05 & & & & 1 & $230.9 \pm 11.9$ & 4.62 \\
\hline 2 & $328.3 \pm 24.5$ & 1.82 & 2 & $427.8 \pm 17.4$ & 0.21 & 2 & $220.8 \pm 9.8$ & 5.22 \\
\hline 4 & $325.0 \pm 20.4$ & 1.88 & 4 & $311.8 \pm 30.3$ & 2.15 & 3 & $266.2 \pm 10.7$ & 3.27 \\
\hline 6 & $282.3 \pm 12.4$ & 2.83 & & & & 4 & $207.2 \pm 9.7$ & 6.64 \\
\hline 8 & $207.2 \pm 9.7$ & 6.64 & & & & & & \\
\hline $\begin{array}{l}\text { Trials } \\
+4 \text { wk SFI } \\
\text { (no.) }\end{array}$ & $\begin{array}{l}\text { Measured } \\
\text { threshold } \\
(\mathrm{sec})^{a}\end{array}$ & $\begin{array}{l}\text { Predicted } \\
\text { trial (no.) })^{b}\end{array}$ & $\begin{array}{l}\text { Trials } \\
+8 \text { wk SFI } \\
\text { (no.) }\end{array}$ & $\begin{array}{l}\text { Measured } \\
\text { threshold } \\
(\mathrm{sec})\end{array}$ & $\begin{array}{l}\text { Predicted } \\
\text { trial (no.) }\end{array}$ & $\begin{array}{l}8 \text { Trials } \\
+ \text { weeks SFI }\end{array}$ & $\begin{array}{l}\text { Measured } \\
\text { threshold } \\
(\mathrm{sec})\end{array}$ & $\begin{array}{l}\text { Predicted } \\
\text { trial (no.) }\end{array}$ \\
\hline \multicolumn{9}{|c|}{ Threshold to first MJ } \\
\hline 1 & $261.4 \pm 17.6$ & 1.58 & & & & 1 & $209.4 \pm 13.2$ & 3.40 \\
\hline 2 & $247.2 \pm 17.9$ & 1.94 & 2 & $256.8 \pm 9.6$ & 1.69 & 2 & $193.3 \pm 10.8$ & 4.54 \\
\hline 4 & $240.8 \pm 7.0$ & 2.12 & 4 & $228.2 \pm 11.6$ & 2.55 & 3 & $232.9 \pm 11.4$ & 2.38 \\
\hline 6 & $246.7 \pm 17.4$ & 1.95 & & & & 4 & $180.0 \pm 9.0$ & 5.95 \\
\hline 8 & $180.0 \pm 9.0$ & 5.95 & & & & & & \\
\hline
\end{tabular}

${ }^{a}$ Mean \pm SEM of the latency (seconds) to the seizure endpoint for the sample.

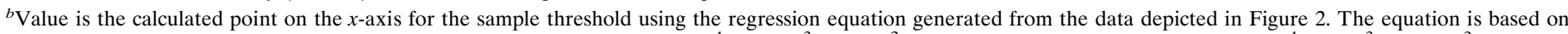

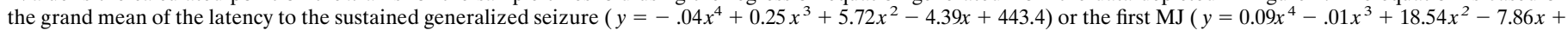
361.74) for all animals on a per-trial basis and is used to estimate the population.

behaviors, when retested at 4 weeks, compared with both the last trial (Table 3) and the groups that had experienced 1, 2, 4, or 6 seizures before the stimulus-free interval $\left(p<0.01, \chi^{2}\right)$. The maximum seizure grade on the retest trial also was found to be influenced by the number of seizures expressed (Table 3). Finally, although there is an apparent trend for the proportion of animals expressing "brainstem seizures" to increase, when retested as a function of the number of pretreatment trials, the median seizure score of the rechallenge trial, however, did not surpass a grade of 2 until eight seizures had been elicited before the 4 week interval (Table 3).

To determine whether the failure to elicit a "brainstem seizure" at 4 weeks after the induction of fewer than eight generalized seizures was a function of the stimulus-free interval, two or four seizures were evoked in separate groups of animals, followed by an 8 week stimulus-free interval. All animals exhibited grade 1 seizures in each trial before the 8 week stimulus-free interval. The behavioral seizure response at 8 weeks was the same compared with the animals that had exhibited 2 or 4 seizures before a 4 week stimulus-free interval. The induction of two generalized seizures resulted in a retest behavioral response profile that was characterized by a median seizure score of 1.5 and a $16.7 \%(1 / 6$, both groups) probability of expressing a "brainstem seizure," regardless of the duration of the stimulus-free interval (4 vs 8 weeks). Similarly, a median seizure score of 2 resulted when animals were retested 4 or 8 weeks after the last of four generalized seizures. Although $33.3 \%$ (2/6) exhibited "brainstem seizure" behaviors at 4 weeks and $16.7 \%(1 / 6)$ expressed this seizure phenotype at 8 weeks, the difference was not significant $\left(\chi^{2}=\right.$ $0.44, p=0.50)$.

\section{Effect of 21 daily trials}

Repeated generalized seizure induction resulted in a progressive decrease in the GST. The reduction was maximal by trial 7 and exhibited no additional decline through trial 21 (Fig. 5). Daily seizure induction also resulted in a progressive increase in the proportion of animals expressing generalized seizure behaviors of grade 3 or higher beginning on trial 11 (Fig. 5). The probability of expressing a "brainstem seizure" by trial 21 was similar to that observed if the animals had experienced eight generalized seizures, and then rechallenged, subsequent to a 2 week stimulusfree period (see Table 2).

\section{DISCUSSION}

This study characterizes a model of epileptogenesis in which the repeated induction of generalized seizure activity elicited by flurothyl modifies the acute and long-term behavioral response of the animal to the convulsant stimulus. Consistent with previous reports, daily exposure to flurothyl was associated with a progressive decrease in both the GST and the latency to the first MJ (Adler et al., 1967; Prichard et al., 1969). The present findings show that although the acute reduction in both seizure thresholds was complete by trial 5 , eight generalized seizures were necessary for the reduction to be long-lasting. The present study also demonstrates that proepileptogenic processes are triggered by eight daily flurothyl-induced generalized seizures and continue to evolve in the absence of additional seizure induction. The longterm changes in neural function that develop during the stimulusfree period were found to be manifest primarily as a qualitative change in the phenotype of the seizure expressed. The degree of change in seizure type was dependent on both the duration of the stimulus-free interval and the number of seizures elicited before this interval.

\section{Changes in seizure threshold}

Altered seizure susceptibility is a well-documented result of repeated seizure activity (Adler et al., 1967; Goddard et al., 1969; Prichard et al., 1969; Mason and Cooper, 1972; Racine, 1972; Cain, 1980; Sangdee et al., 1982). In the present study, the expression of a single generalized clonic seizure was sufficient to 


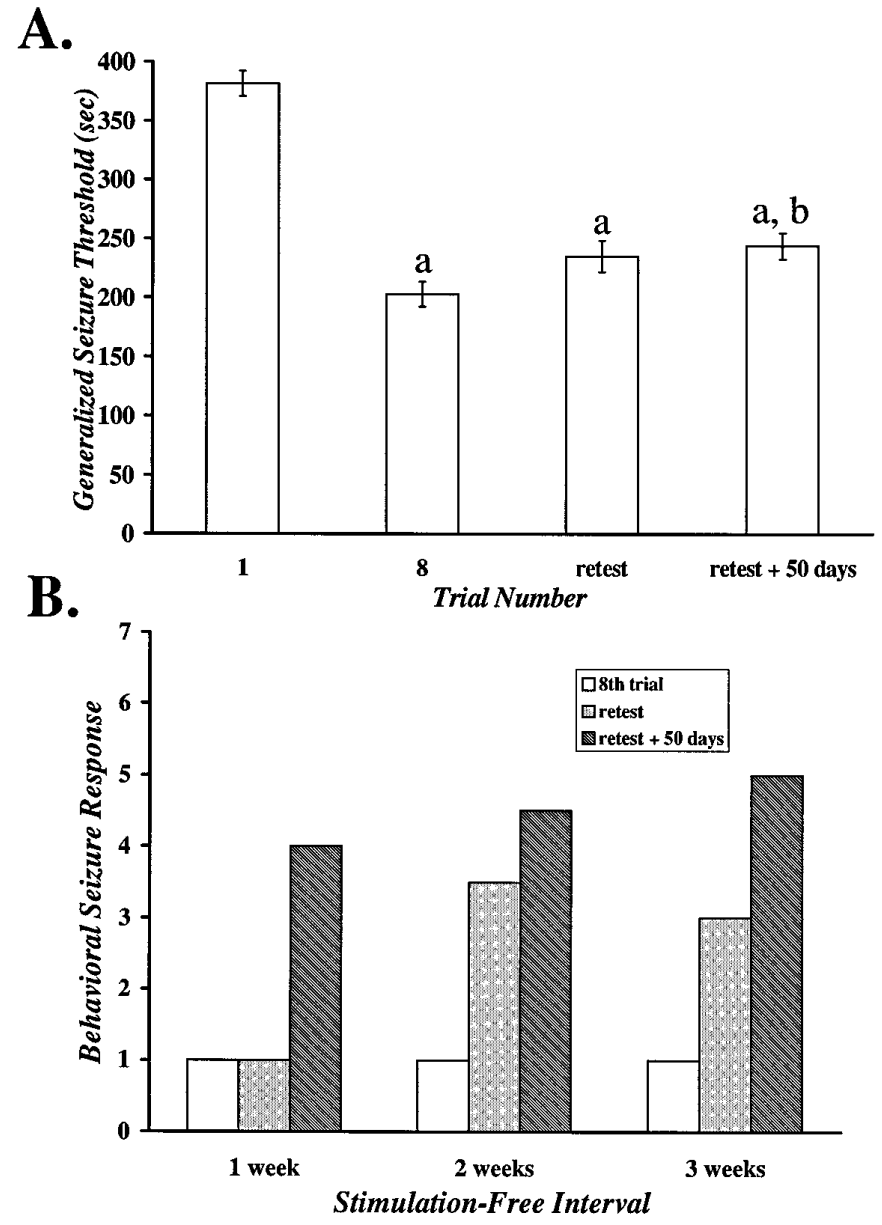

Figure 3. The effect of 50 additional stimulus-free days after the retest trial on the GST $(A)$ and the behavioral seizure response $(B) . A$, The GST is significantly lower compared with the naive state (trial 1$)$ up to $50 \mathrm{~d}$ after the retest trial. Data are the mean \pm SEM of a subset of animals rechallenged $50 \mathrm{~d}$ after the retest trial, which followed a 1,2 , or 3 week stimulation-free interval that was preceded by eight daily seizures $(n=$ 14); $a, p<0.001$ versus trial $1 ; b, p<0.05$ versus trial 8 . $B$, The behavioral seizure response continues to change during an additional $50 \mathrm{~d}$ stimulation-free interval. Data are the median seizure score of the animals in $A$ ( $n=5,6,3 ; 1,2,3$ weeks, respectively).

produce a significant reduction in the latency to both the first MJ and the sustained generalized seizure when retested $24 \mathrm{hr}$ later. Both thresholds continued to decline with each subsequent trial until trials 4-5, at which time, no additional changes were observed. These threshold profiles suggest that the neural mechanism(s) responsible for the increase in seizure susceptibility can be induced by a single seizure event. Moreover, the processes responsible are apparently reinforced with each additional seizure until a threshold "floor" is reached at the fifth trial. Because flurothyl does not depend on metabolism, either for its action or for its elimination, and because the concentration of flurothyl in brain after inhalation is not altered by repeated exposure (Gallagher, 1969), the change in seizure threshold is most likely attributable to repeated ictal activity rather than to changes in the pharmacokinetics of the drug.

The number of seizures was an important factor in determining whether decreases in seizure threshold were maintained over a stimulus-free interval. Despite the fact that by the fifth trial, seizure thresholds for both the first MJ and the GST were max- imally reduced, this was not sufficient for the change to be long-lasting. For example, the expression of six generalized seizures resulted in a significant and maximal reduction in the GST. However, six seizures were not sufficient to induce a long-lasting, maximal change in the GST. Maximal threshold changes were maintained only after the expression of eight seizures. These data suggest that a minimum degree of input is necessary to reinforce alterations in neuronal excitability, such that the modified susceptibility is long-lasting.

An unexpected outcome of the threshold analysis was the observed variability in seizure thresholds measured after different stimulus-free intervals. Although the GST was consistently lower on the retest trial in all groups of mice compared with naive animals, there was partial recovery of the GST in all groups. The most pronounced recovery was observed after a 3 week stimulusfree interval. At this time point, both thresholds had recovered to a level equivalent to trial 3 . This partial recovery was apparently real, because two independent replications were performed with separate groups of animals represented at each time point. These data suggest that there are active processes that attempt to reestablish the basal threshold. The fact that the threshold, after a 4 week stimulus-free interval, was nearly identical to that observed on the last of eight trials indicates that these processes either fail or are in a state of continuous flux. Regardless, it is clear that the GST is permanently altered compared with the naive state, because animals tested $50 \mathrm{~d}$ after a specified stimulusfree interval presented with a GST significantly different from that observed on the first trial.

\section{Changes in seizure behavior}

Terminating the administration of flurothyl and opening the chamber with the beginning of sustained motor seizure activity resulted in the expression of predominantly generalized clonic seizure behaviors over the course of eight daily trials. However, after a 2-4 week stimulus-free interval, a different behavioral response resulted when the animal was retested. This response was characterized first by the expression of a generalized clonic seizure lasting $\sim 15-20 \mathrm{sec}$, followed by the progression, without interruption, to a qualitatively distinct set of motor behaviors consisting of an initial running/bouncing episode, and the progression to seizures with tonic manifestations. This progression was stereotyped, in that generalized clonic seizure behaviors always preceded running/bouncing seizure events, which in turn always preceded the expression of forelimb/hindlimb treading or seizures with tonic components (see Fig. $1 B$ ). In a naive animal, this progression from clonic to running/bouncing to tonic seizures is rarely seen using our protocols.

The motor components of generalized seizures in experimental animals have been shown to be associated with specific anatomical systems. Whereas generalized clonic seizures require the integrity of forebrain structures for their expression (Browning and Nelson, 1986; Magistris et al., 1988; Browning et al., 1993), brainstem circuitry is both necessary and sufficient for the expression of running/bouncing and tonic motor manifestations (Kreindler et al., 1958; Browning and Nelson, 1986). Thus, the progression of flurothyl-induced seizure behaviors seen after exposure to our paradigm appears to be the result of an initial activation of the forebrain system followed rapidly by activation of the brainstem seizure system. This finding suggests that the epileptogenic processes triggered by the repeated induction of generalized clonic seizure activity alter neural systems such that recruitment of brainstem circuitry by the forebrain is facilitated. 
Table 2. Effect of the duration of the stimulus-free interval on the behavioral seizure response

Animals with behavioral seizure score $\geq$ grade $3(\%)^{b}$

\begin{tabular}{|c|c|c|c|c|c|c|c|}
\hline Stimulus-free interval $^{a}$ & $n$ & First trial & Last trial & Retest & First trial & Last trial & Retest \\
\hline 1 week & 13 & 0.0 & 15.4 & 15.4 & 1 & 1 & 1 \\
\hline 2 weeks & 24 & 0.0 & 0.0 & $50.0^{* * *}$ & 1 & 1 & $2.5^{* * *}$ \\
\hline 3 weeks & 11 & 9.1 & 0.0 & $63.6^{*}$ & 1 & 1 & $3 * * *$ \\
\hline 4 weeks & 18 & 5.6 & 11.1 & $88.9^{* * * *}$ & 1 & 1 & $4^{* * *}$ \\
\hline
\end{tabular}

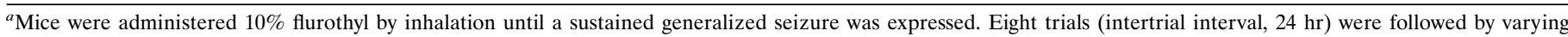
periods of nonexposure to the convulsant stimulus when the mouse was retested and the behavioral seizure score assigned.

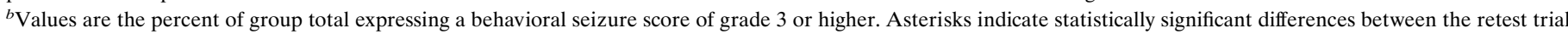
and the last trial by the McNemar test; ${ }^{*} p<0.05,{ }^{* *} p<0.005,{ }^{* * * *} p<0.001$.

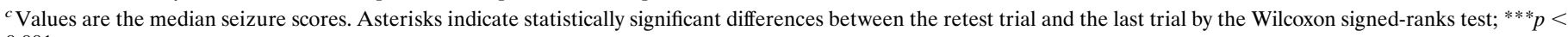
0.001 .

Figure 4. Correlational analysis indicating a lack of association between the GST and the expression of a specific generalized seizure behavior on retest. Threshold and behavioral measures were made after a 1 (solid circles), 2 (solid squares), 3 (solid triangles), or 4 (solid diamonds) week stimulus-free interval, which was preceded by an induction phase of eight consecutive daily seizures (Spearman's $\rho=-0.092$ ).

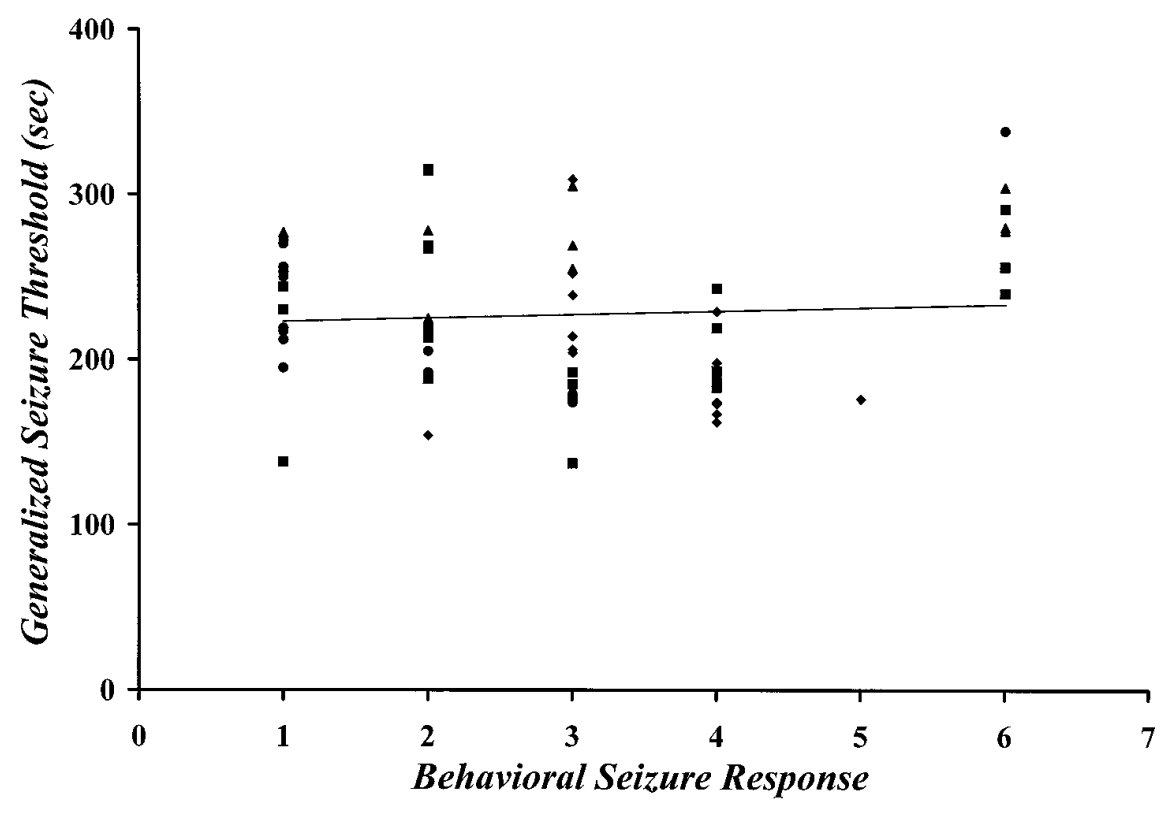

Table 3. Effect of the number of generalized seizures on the behavioral seizure response

Animals with behavioral seizure score $\geq \operatorname{grade} 3(\%)^{b}$

\begin{tabular}{|c|c|c|c|c|c|c|c|}
\hline No. of seizures ${ }^{a}$ & $n$ & First trial & Last trial & Retest & First trial & Last trial & Retest \\
\hline 1 & 5 & 0.0 & XXx & 0.0 & 1 & 1 & 1 \\
\hline 2 & 6 & 0.0 & 0.0 & 16.7 & 1 & 1 & 1.5 \\
\hline 4 & 6 & 0.0 & 0.0 & 33.3 & 1 & 1 & $2 *$ \\
\hline 6 & 6 & 0.0 & 0.0 & 33.3 & 1 & 1 & $2 *$ \\
\hline 8 & 18 & 5.6 & 11.1 & $88.9 * * * *$ & 1 & 1 & $4 * * *$ \\
\hline
\end{tabular}

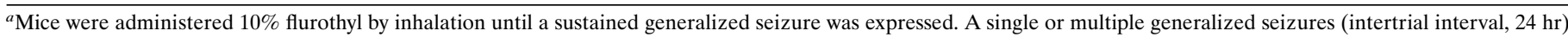
were followed $28 \mathrm{~d}$ later by a retest trial when the behavioral seizure score was assigned.

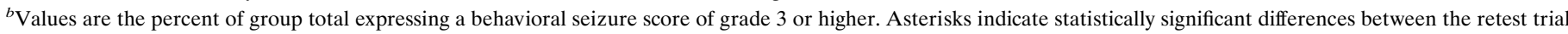
and the last trial by the McNemar test: ${ }^{* * *} p<0.001$.

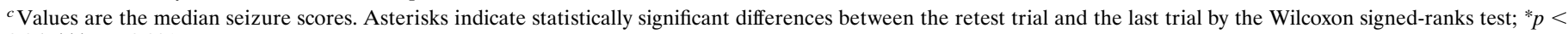
$0.05, * * * p<0.001$.

The mechanism by which facilitated recruitment of the brainstem seizure system occurs, after exposure to our paradigm, is unknown. However, this effect could be mediated by (1) a lowering of the threshold for activation of the brainstem seizure system, (2) facilitated propagation of seizure activity between the forebrain and brainstem systems, or (3) a combination of facilitated propagation and lowered brainstem seizure system threshold. Which of these possibilities is correct has major implications for concepts of the organization of seizure expression systems in the brain and the effects of repeated seizure activity on those systems. For example, if exposure to our paradigm simply lowers the threshold for activation of the brainstem seizure system, then forebrain seizure activity must always access the brainstem system. In the seizure-naive state, however, brainstem seizures are not expressed, because forebrain-driven discharge fails to exceed the threshold for initiating seizure activity in the brainstem. By 


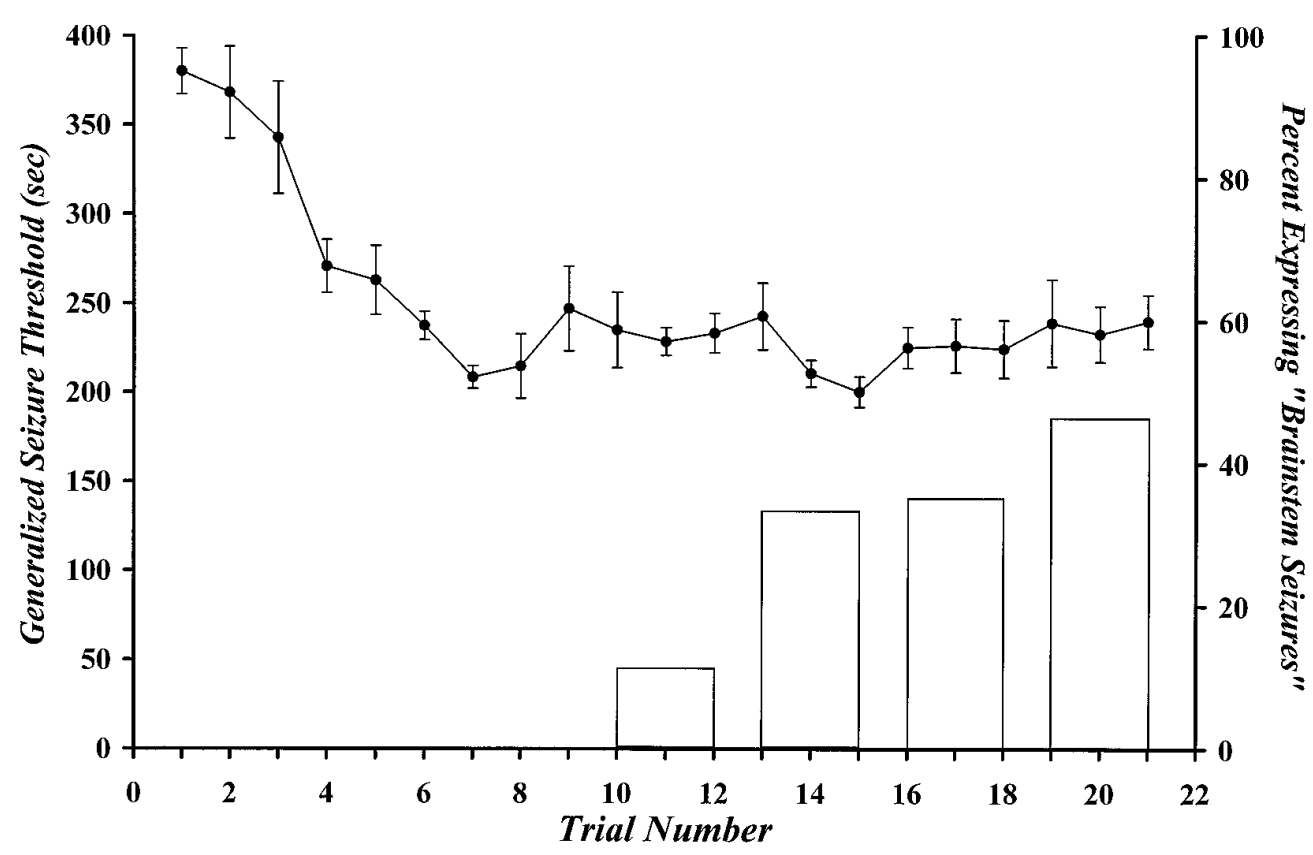

Figure 5. Effect of 21 consecutive daily flurothyl-induced generalized seizures on the GST and the generalized seizure phenotype. Points represent the mean GST \pm SEM of six animals. The bars represent the proportion of generalized seizures that were "brainstem seizures" within a defined "bin." A bin is composed of the total number of seizures in regular three trial intervals. Continuing stimulation for 2 weeks beyond the first eight trials did not significantly alter the proportion of mice expressing "brainstem seizures" (cf. Table 2). contrast, if facilitated propagation from the forebrain to the brainstem is solely responsible for the change in phenotype in our model, then exposure to our paradigm would be conceived of as promoting a fundamental change in the interaction between the forebrain and brainstem seizure systems; that is, in the seizurenaive state, these systems are independent, and seizure activity elicited in either system is confined to that system. After exposure to our paradigm, however, the propagation of forebrain seizure activity to the brainstem system is facilitated through the establishment of a new propagation network. Experiments are currently being conducted to establish the basis for the reliable change in seizure phenotype observed in our model.

The finding that a significant shift in the behavioral response occurs when an animal is retested after a stimulus-free interval indicates that seizure-induced changes within the brain continue to evolve independent of additional stimulation. However, the progression from a purely generalized clonic seizure to a seizure consisting of "brainstem seizure" behaviors was not observed in all animals when retested after the stimulus-free interval. Instead, the probability of this occurrence and the phenotype of the seizure were found to be associated with both the duration of the stimulus-free interval that preceded the retest trial and the number of seizures induced before this interval. A significant increase in the number of animals exhibiting behaviors of grade 3 or above was observed only if the animal had experienced eight generalized seizures and at least a 2 week stimulus-free interval before retesting. By contrast, fewer than eight seizures did not affect the behavioral response compared with the last trial, regardless of whether the duration of the stimulus-free interval was 4 or 8 weeks. These data suggest that a minimum of seven or eight generalized seizure events are necessary to elicit a change in the behavioral seizure response. This minimum appears to be an absolute, because fewer than eight seizures did not reliably result in a change in seizure type, whether given 4 or 8 weeks to develop. Furthermore, once induced, the epileptogenic processes progressively develop in the absence of continued input, such that the probability of exhibiting the behavioral progression is directly related to the duration of the stimulus-free interval.
Other lines of evidence suggest that generalized seizure activity induces changes within the brain that are time-dependent and develop in the absence of additional input. For example, cytochrome oxidase activity is unchanged $24 \mathrm{hr}$ after the last of a series of eight generalized electroconvulsive shock seizures, but is increased in a number of brain areas $28 \mathrm{~d}$ after the last seizure (Nobrega et al., 1993). Moreover, a single electroshock stimulus, when followed by a treatment-free interval, is as effective in reducing nigral dopamine autoreceptor subsensitivity as is the administration of repeated electroshock seizures (Chiodo and Antelman, 1980). Furthermore, clinical studies have shown that on the recurrence of seizure activity after a "silent interval," patients present with more complex behavioral manifestations than before the seizure-free interval (French et al., 1993). Together, these data demonstrate that some of the long-term consequences associated with seizure activity develop during a period in which overt clinical seizures are not evident.

An intriguing result of the present study is the observation that the mechanism(s) responsible for the change in seizure phenotype occurs equally in the presence or absence of continued daily seizure induction past trial 8 . This raises the possibility that animals allowed to have a stimulus-free period before retesting experienced spontaneous ictal activity. During the times when mice were periodically monitored, over the course of the stimulus-free interval, we have never observed any overt motor seizure activity. This does not preclude the possibility that spontaneous subclinical seizure activity occurred during this interval. Regardless, this study has demonstrated that in either case, the mechanism(s) involved requires a minimum of eight generalized seizures to be triggered, which then progressively evolve over a defined time frame.

\section{GST and seizure phenotype}

Exposure to our paradigm initiates a process that results in a lowering of GST acutely, which is then variably maintained in a lowered state for at least $71 \mathrm{~d}$. Exposure to our paradigm also initiates a process that evolves only slowly over time and that promotes a change in the final type of seizure expressed by the 
animal. Correlational analyses indicate a lack of association between measures of GST and the change in seizure phenotype. This outcome suggests that the underlying processes mediating the reduction in GST may be independent from the processes underlying the change in seizure phenotype from clonic to tonic.

Because the initial seizure manifestation in response to flurothyl is always clonic, the latency to clonic seizure onset represents a measure of the threshold for the forebrain seizure system. Repeated flurothyl exposure has been reported to lower the focal forebrain afterdischarge thresholds of both the amygdala and the frontal cortex and to facilitate the rate of electrical kindling from both of these structures (Okada et al., 1985; Wong and Moshe, 1987). We have observed identical effects for olfactory bulb kindling in mice preexposed to our paradigm (our unpublished observations). These data suggest that repeated flurothyl-induced seizures not only lower forebrain seizure threshold but also facilitate seizure propagation within the forebrain. Thus, within the forebrain seizure system, exposure to our paradigm promotes apparently identical effects to those seen after electrical kindling from the limbic system. In addition, the outcome of a recent pharmacological study from our laboratory indicates that drugs effective at blocking the development of electrical kindling also effectively block the change in seizure phenotype observed after exposure to our model (Applegate et al., 1997). These data indicate that the synaptic mechanisms underlying epileptogenic processes in electrical kindling and our model system share considerable overlap.

Whether repeated flurothyl-induced seizures lower the threshold and/or facilitates propagation in the brainstem seizure system is currently unknown. However, previous amygdala kindling to five consecutive stage 5 seizures has been shown to increase the probability of tonic hindlimb extension after corneal electroshock stimulation (Applegate et al., 1991). These data suggest that repeated forebrain afterdischarge activity can significantly influence the brainstem seizure system. Experiments are currently underway to elucidate further the mechanisms through which these effects are mediated.

In summary, this study describes a model of epileptogenesis in which the repeated induction of generalized clonic seizure activity results in time-dependent changes in neural function that can evolve in the absence of continued seizure induction. Initial alterations involve reductions in the seizure threshold. Mechanisms that require more time to develop and, once initiated, can evolve in the absence of additional input, are manifest primarily as an erosion of the separation between the two anatomical systems that mediate generalized seizure behaviors. Thus, the barrier that prevents "forebrain seizure" activity from recruiting "brainstem seizure" systems is altered in such a way as to allow the expression of "brainstem seizure" behaviors. Because the process that promotes changes in the behavioral response of the animal occurs in the absence of continued seizure induction, this model may be useful in studying the mechanisms that underlie increases in seizure susceptibility and the development of motor seizures, without the confounding influence of an ictal event.

\section{REFERENCES}

Adler MW, Sagel S, Kitagawa S, Segawa T, Maynert EW (1967) The effects of repeated flurothyl-induced seizures on convulsive thresholds and brain monoamines in rats. Arch Int Pharmacodyn Ther 170:12-21.
Applegate CD, Samoriski GM, Burchfiel JL (1991) Evidence for the interaction of brainstem systems mediating seizure expression in kindling and electroconvulsive shock seizure models. Epilepsy Res 10:142-147.

Applegate CD, Samoriski GM, Odzduman K (1997) Effects of valproate, phenytoin and MK801 in a novel model of epileptogenesis. Epilepsia, 38:631-637.

Browning RA, Nelson DK (1986) Modification of electroshock and pentylenetetrazol seizure patterns in rats after precollicular transections. Exp Neurol 93:546-556.

Browning RA, Maggio R, Sahibzada N, Gale K (1993) Role of brainstem structures in seizures initiated from the deep prepiriform cortex of rats. Epilepsia 34:393-407.

Buterbaugh GG, Hudson GM (1990) The kindling process and vulnerability to status epilepticus. In: Kindling 4 (Wada JA, ed), pp 169-183. New York: Plenum.

Cain DP (1980) Effects of kindling or brain stimulation on pentylenetetrazol-induced convulsion susceptibility. Epilepsia 21:243-249.

Chiodo LA, Antelman SM (1980) Electroconvulsive shock: progressive dopamine autoreceptor subsensitivity independent of repeated treatment. Science 210:799-801.

Davis WM, Webb OL (1963) A circadian rhythm of chemoconvulsant response thresholds in mice. Med Exp 9:263-267.

Dennison Z, Teskey GC, Cain DP (1995) Persistence of kindling: effect of partial kindling, retention interval, kindling site, and stimulation parameters. Epilepsy Res 21:171-182.

French JA, Williamson PD, Thadani VM, Darcey TM, Mattson RH, Spencer SS, Spencer DD (1993) Characteristics of medial temporal lobe epilepsy. I. Results of history and physical examination. Ann Neurol 34:774-780.

Gallagher BB (1969) Seizure threshold and hexafluorodiethyl ether in brain tissue. Biochem Pharmacol 18:542-544.

Goddard GV, McIntyre DC, Leech CK (1969) A permanent change in brain function resulting from daily electrical stimulation. Exp Neurol 25:295-330.

Kreindler A, Zuckermann E, Steriade M, Chimion D (1958) Electroclinical features of convulsions induced by stimulation of brain stem. J Neurophysiol 21:430-436.

Lothman EW, Bertram EH, Kapur J, Stringer JL (1990) Recurrent spontaneous hippocampal seizures in the rat as a chronic sequela to limbic status epilepticus. Epilepsy Res 6:110-118.

Magistris MR, Mouradian MS, Gloor P (1988) Generalized convulsions induced by pentylenetetrazol in the cat: participation of forebrain, brainstem, and spinal cord. Epilepsia 29:379-388.

Mason CR, Cooper RM (1972) A permanent change in convulsive threshold in normal and brain damaged rats with repeated small doses of pentylenetetrazol. Epilepsia 13:663-674.

National Institutes of Health (1985) Guide to the care and use of laboratory animals. Publication No. 85-23 (Rev). Bethesda, MD: NIH.

Nobrega JN, Raymond R, DiStefano L, Burnham WM (1993) Longterm changes in regional brain cytochrome oxidase activity induced by electroconvulsive treatment in rats. Brain Res 605:1-8.

Okada R, Moshe SL, Ono K, Albala BJ (1985) Unidirectional interaction between flurothyl seizures and amygdala kindling. Brain Res 344:103-108.

Prichard JW, Gallagher BB, Glaser GH (1969) Experimental seizure threshold testing with flurothyl. J Pharmacol Exp Ther 166:170-178.

Racine RJ (1972) Modification of seizure activity by electrical stimulation. II. Motor seizure. Electroencephalogr Clin Neurophysiol 32:281-294.

Ramer D, Pinel JP (1976) Progressive intensification of motor seizures produced by periodic electroconvulsive shock. Exp Neurol 51:421-433.

Sangdee P, Turkanis SA, Karler R (1982) Kindling-like effect induced by repeated corneal electroshock in mice. Epilepsia 23:471-479.

Truitt Jr EB, Ebersberger EM, Ling ASC (1960) Measurement of brain excitability by use of hexafluorodiethyl ether (Indoklon). J Pharmacol Exp Ther 129:445-453.

Wong BY, Moshe SL (1987) Mutual interactions between repeated flurothyl convulsions and electrical kindling. Epilepsy Res 1:159-164. 